\title{
Habituation as a function of similarity and temporal location of target and distractor stimuli
}

\author{
HELEN KAYE, NIGEL SWIETALSKI, and N. J. MACKINTOSH \\ University of Cambridge, Cambridge, England
}

\begin{abstract}
Three experiments examined the habituation of rats' neophobia to novel flavors, and the disruption of that habituation by presentation of a distractor flavor either immediately before or immediately after the target flavor. Habituation of neophobia to lemon solution was more seriously disrupted by presentation of saline as a distractor than by presentation of coffee as a distractor, and this was true whether the distractor was presented before or after the target on each habituation trial. Two further experiments established that the relative ineffectiveness of coffee as a distractor could not be attributed to its lack of salience, and was probably related to its greater similarity to the target lemon flavor. These results do not fully accord with those reported by Robertson and Garrud (1983), but are readily explained in terms of generalization of habituation between distractor and target flavors.
\end{abstract}

Repeated presentation of a target stimulus usually results in a decline in, or habituation of, the responses it initially elicited. But such habituation may be disrupted by the presentation of another novel stimulus or distractor (e.g., Thompson \& Spencer, 1966). Thompson and Spencer argued that this disruption, or dishabituation, was really no more than a matter of sensitization: the presentation of the novel distractor momentarily raises the animal's level of arousal and increases responsiveness to the next presentation of the habituated target stimulus. Their analysis finds support in their demonstration that the effect of the distractor may be extremely transient: responding to the habituated stimulus is increased if it is presented immediately after the distractor but not if it is presented a minute or so later.

Other studies, however, have demonstrated distractor effects that cannot be interpreted in this way. Rats offered a novel-tasting fluid will drink relatively little on first exposure, but this neophobia habituates with repeated exposure to the taste. Distractor effects with this preparation can be produced by offering the rats a second novel substance to drink immediately after the first, even though the interval between habituation trials, and therefore the interval between distractor and subsequent presentation of the habituated flavor, is several hours (e.g., Green \& Parker, 1975; Robertson \& Garrud, 1983; Shanks, Preston, \& Stanhope, 1986). The distractor flavor can hardly increase the rat's level of arousal on a test trial that may occur up to $24 \mathrm{~h}$ later, and, in Green and Parker's (1975) experiment, the effect of the distractor was more marked the more closely it followed the preced-

This research was supported by a grant from the United Kingdom Science and Engineering Research Council to N. J. Mackintosh. Requests for reprints should be sent to the first author, at the Department of Experimental Psychology, University of Cambridge, Downing Street, Cambridge CB2 3EB, England. ing trial rather than the more closely it preceded the following trial. All of these investigators therefore have interpreted their results in terms of disruption of memory. Habituation of neophobia, they have suggested, depends on having a representation of the target flavor in memory at the time of the test trial. Green and Parker (1975) argued that the novel distractor interfered with consolidation of this memory, thus explaining why the effect of the distractor declined as the interval between target and distraction increased, whereas Robertson and Garrud (1983) and Shanks et al. (1986) interpreted their results in terms of Wagner's $(1976,1981)$ theory of memory and habituation.

According to Wagner, habituation occurs because a stimulus fails to gain access to a limited-capacity processor; this will happen whenever a representation of that stimulus is already present in short-term memory at the moment when the stimulus is presented. Habituation may be disrupted by presentation of a novel distractor immediately after the target stimulus because the distractor will displace the represenation of the target stimulus from the limited-capacity processor and thus reduce the probability that the target will be represented in short-term memory on the next trial.

Robertson and Garrud (1983) reported two further results which they interpreted in terms of Wagner's account. First, they found that although habituation to sucrose solution was disrupted by presentation of coffee flavor as a distractor, habituation to lemon flavor was not. Independent evidence suggested that lemon and coffee were rather similar to the rat, but that sucrose and coffee were not, and they thus concluded that only if the distractor was quite different from the target stimulus would it disrupt habituation to the target. They argued that Wagner's theory must predict that a distractor similar to a recently presented target will fail to gain access to the processor and therefore cannot act as an effective distractor. The 
explanation will clearly account for the results they obtained but seems unnecessarily complicated. In the limiting case in which distractor and target are identical, that is, the case in which the experimenter presents the same target stimulus in rapid succession, it is hard to see how the second presentation of the stimulus could do anything other than increase habituation produced by the first presentation. Even if target and distractor are not identical but only similar, generalization of habituation from distractor to target could easily counteract the otherwise disruptive effects its presentation might have.

The second result reported by Robertson and Garrud (1983) is more surprising. In a subsequent experiment, they found that when the distractor was presented immediately before the target stimulus on a habituation trial, the effect of similarity between distractor and target was precisely opposite what they had observed when distractor followed target. That is to say, presentation of sucrose as distractor immediately before coffee did not disrupt habituation to coffee, but presentation of the similar lemon flavor as distractor did disrupt habituation. Their interpretation was as follows. Presentation of a different distractor after the target disrupts habituation to the target by displacing its representation from the processor. But presentation of a different distractor before the target will have no effect on habituation because it is now the target that displaces the distractor. Presentation of a similar distractor after the target has no effect on habituation because it cannot gain access to the processor; but presentation of a similar distractor before the target will disrupt habituation because it is now the distractor that prevents the target from gaining access to the processor. Once, again, we are not wholly convinced by this explanation, for it ignores the extent to which habituation to the distractor will generalize to the target stimulus. In the limiting case in which target and distractor are identical there would be perfect generalization between them and therefore enhanced habituation.

Our initial aim was to replicate Robertson and Garrud's (1983) results. Specifically, we wished to see whether a single experiment could demonstrate that a similar distractor would disrupt habituation to a target if presented before the target but not if presented after it, whereas a dissimilar distractor would disrupt habituation if presented after the target but not if presented before.

\section{EXPERIMENT 1}

The three solutions used by Robertson and Garnud (1983) in their experiments were $3 \%$ lemon, $1.25 \%$ coffee, and $5 \%$ sucrose. They gave rats a single habituation trial followed $6 \mathrm{~h}$ later by a 10-min test trial. On the habituation trial, the target and distractor flavors were available either for $5 \mathrm{~min}$ each or in a fixed quantity $(1.5$ or $2.0 \mathrm{ml})$. The procedures employed in the present experiments differed in two major (as well as several minor) ways. First, we used a 3\% saline solution instead of sucrose. There is no reason to believe that saline is not also different from lemon and coffee flavors, and it has the advantage over sucrose of being less preferred: Robertson and Garrud's rats drank nearly twice as much sucrose as lemon in a 5-min period, and this preference raises unwelcome possibilities of contrast effects, as well as other potential problems. Secondly, we gave a series of three habituation trials, with or without a distractor, spaced $24 \mathrm{~h}$ apart, before a final test trial $24 \mathrm{~h}$ later, with the target flavor being available either for $10 \mathrm{~min}$ on each trial or until the rat had consumed $5 \mathrm{ml}$. In a series of pilot experiments, we found little evidence either of habituation after a single presentation of the lemon flavor or that a dissimilar distractor presented after the lemon had any effect on its subsequent consumption. This was true whether the other parameters of the experiments were similar to those employed by Robertson and Garrud or not. The training data of Experiment 1 confirm that habituation and distractor effects took more than one trial to develop.

In Experiment 1, we asked whether a dissimilar distractor (saline) presented after a target solution (lemon) would disrupt habituation of neophobia to the target, while another, presumably more similar, distractor (coffee) would not.

\section{Method}

Subjects and Apparatus. The subjects were 30 male hooded Lister rats, approximately 4-6 months old at the start of the experiment. They had previously been used in an experiment in which they licked at water tubes in an operant chamber, and had been maintained on a water-deprivation schedule of 30 -min access to water in their home cages after the end of that experiment. Throughout the present experiment, they received 20 -min access to water in their home cages after their daily sessions.

The apparatus consisted of eight plastic mouse cages with grid ceilings. A metal drinking tube, attached to a $50-\mathrm{ml}$ calibrated glass cylinder, protruded through one end wall.

Procedure. On Days 1-2, the animals received 15-min access to water in the experimental apparatus, and on Day 3 they were divided into three groups of 10 . On Days 3, 4, and 5, all animals received 10-min access to a $3 \%(\mathrm{v} / \mathrm{v})$ lemon solution (Borden Normal Strength lemon juice). Animals in Group $L$ were then returned to their home cages immediately after drinking the lemon solution each day. The remaining two groups were given 10 -min access to a distractor solution before being returned to their home cages. For Group L-S, the distractor was $3 \%(w / v)$ saline solution $(\mathrm{NaCl})$; for Group L-C, it was $1.5 \%(w / v)$ coffee (Hag Instant Decaffeinated). On Day 6, the final test day, all animals received 10-min access to the lemon solution.

\section{Results and Discussion}

The three groups were matched for their consumption of lemon solution on Day 3 (the first day of habituation); Group L drank $5.7 \mathrm{ml}$, Group L-S, $5.9 \mathrm{ml}$, and Group $\mathrm{L}-\mathrm{C}, 6.1 \mathrm{ml}$ of lemon solution.

Consumption of the lemon solution on all 3 training days and in the final test session is shown in Figure 1. Over the first 2 training days there does not appear to be any difference between the groups, but by the 3rd day, Group $L$ increased its consumption of lemon, that is, was showing habituation; this increase was matched by Group 


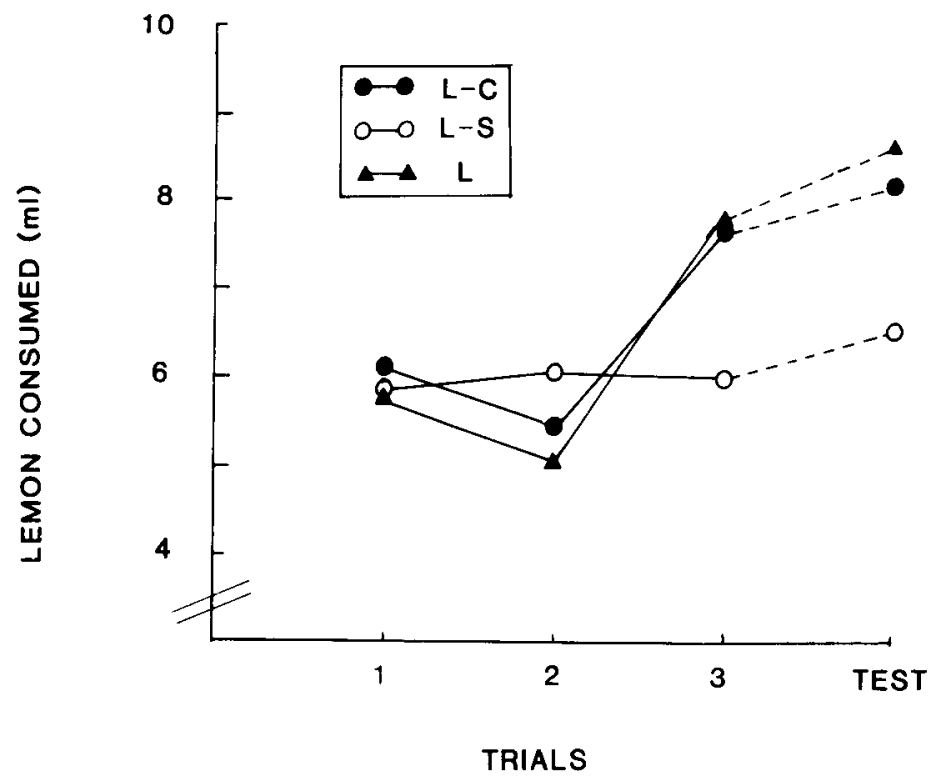

Figure 1. Results of Experiment 1. Consumption of the lemon solution on each day of habituation training and on the final test day.

L-C but not by Group L-S. These differences were maintained on the final test day, with the results showing that habituation of neophobia to lemon was disrupted in Group L-S but not in Group L-C. Training and test days were analyzed separately. An analysis of variance on the first 3 days of habituation established that there was a significant increase in the consimption of lemon $[F(2,54)=$ $9.56, p<.01]$ but no reliable difference between groups $(F<1)$; the interaction between days and groups fell just short of significance $[F(4,54)=2.52, .10>p>.05]$. But an analysis of variance on the test data established that the three groups differed in their consumption of lemon on this day $[F(2,27)=5.44, p<.05]$, and subsequent Newman-Keuls tests showed that Group L-S drank significantly less lemon that did either Group L or Group L-C, which did not differ from one another.

These results confirm, in a single experiment, the first main conclusion drawn by Robertson and Garrud (1983) from separate experiments. Habituation of neophobia to lemon was disrupted by exposure to one distractor, saline, immediately after each habituation trial, but not by exposure to another distractor, coffee, presumed to be more similar to the lemon solution. There are several minor discrepancies between our results and theirs. First, Robertson and Garrud, like others (Green \& Parker, 1975; Shanks et al., 1986), observed both habituation and distractor effects after a single trial. As we have already noted, in our experiment habituation of neophobia to lemon developed relatively slowly and there was no suggestion of a distractor effect after one habituation trial. We do not have any obvious explanation for this discrepancy: it is unlikely to be due to differences in the interval between habituation and test trials, or in our choice of saline rather than sucrose as distractor, since pilot experiments that had followed Robertson and Garrud's pro- cedures more closely were equally unsuccessful. A second discrepancy is that in Robertson and Garrud's (1983) Experiment 1, animals given coffee as a distractor after drinking lemon actually drank more lemon on test than did their control group. The most plausible explanation for this discrepancy is that the particular coffee and lemon flavors we added to the water did not taste quite the same to our rats as Robertson and Garrud's solutions did to theirs. Indeed, the coffee solution we used was slightly stronger than theirs (1.5\% against $1.25 \%)$, largely because our pilot experiments had failed to find any increase in consumption of lemon using $1.25 \%$ or $1.0 \%$ coffee solution as distractor. But neither the discrepancy nor its explanation seem particularly important. The relevant conclusion is that coffee is less effective in disrupting habituation to lemon than is saline when the distractor solutions are presented after the target.

\section{EXPERIMENT 2}

Experiment 2 asked whether, as Robertson and Garrud's (1983) results would imply, coffee would be more effective than saline at disrupting habituation of neophobia to lemon when the distractors were presented before the target solution. In this experiment, since we did not wish the animals to have consumed different amounts of fluid immediately before being offered lemon solution to drink, the control group received water before lemon and all groups were given a fixed quantity of distractor to drink immediately before their free access to lemon on each training day.

\section{Method}

Subjects and Apparatus. The subjects were 18 male hooded Lister rats of similar age and prior experience to those used in Ex- 
periment 1 . The apparatus was exactly the same, except that the distractor solutions were presented in small glass tubes that were filled with exactly $2 \mathrm{ml}$ of fluid.

Procedure. In all unspecified respects, the general procedure was the same as in Experiment 1. After 2 days of drinking water from the metal tubes, the animals received a 3rd pretraining day on which they drank $2 \mathrm{ml}$ of water from the glass tubes subsequently used to present the distractor. On Days 4,5, and 6, the rats were divided into three groups of 6 and given $2 \mathrm{ml}$ of tap water (Group W-L) $3 \%$ saline (Group S-L), or $1.5 \%$ coffee (Group C-L) to drink before their $10-\mathrm{min}$ access to $3 \%$ lemon solution. On Day 7 , they were all given 10 -min access to lemon without prior presentation of the distractor.

\section{Results and Discussion}

The groups were matched for consumption of water on Day 2. Their scores were: Group W-L, $8.8 \mathrm{ml}$; Group S-L, $9.8 \mathrm{ml}$; and Group C-L, $8.7 \mathrm{ml}$. Consumption of the lemon solution on the 3 days of habituation training and on the final test day is shown in Figure 2.

By controlling consumption of the distractor before giving access to the lemon solution, we had hoped that the three groups would drink comparable amounts of lemon, at least on the 1st day of training. Figure 2 shows how wrong we were: the prior consumption of saline solution appears to have significantly increased consumption of lemon on all 3 training days. An analysis of variance confirmed this. There was an overall increase in consumption of lemon over the 3 days $[F(2,30)=15.15, p<.01]$, a significant difference between the three groups $[F(2,15)$ $=5.66, p<.05]$, and no interaction between days and groups $(F<1)$. A contrast analysis showed that, over the 3 days combined, Groups W-L and C-L drank the same amount of lemon $(F<1)$, but the two groups together drank significantly less than Group S-L $[F(1,15)$ $=10.73, p<.01]$.

The test trial, on which the animals were offered the lemon solution alone to drink, revealed a different pic- ture. It is apparent that both Group S-L and Group C-L drank less lemon than Group W-L. An analysis of variance revealed a significant group effect $[F(2,15)=5.63$, $p<.05]$ and Newman-Keuls tests revealed that Group W-L differed from each of the other groups, which did not differ from one another.

These results do not accord with those of Robertson and Garrud (1983). It is true that coffee as a distractor, which failed to disrupt habituation to lemon when presented after lemon, did disrupt habituation when presented before lemon. This conforms to their conclusions. But they reported that a dissimilar distractor, which disrupted habituation when presented after the target flavor, had no effect when presented before, whereas in the present experiments the presumably dissimilar saline solution disrupted habituation to lemon regardless of whether it was presented before or after lemon. Indeed, it is probable that the present experiment underestimates the disruption of habituation produced by prior presentation of saline. Since Group S-L drank more lemon on habituation trials than did the other groups, they presumably had greater opportunity to habituation their neophobia. They nevertheless drank less lemon on test than did either of the other groups.

\section{EXPERIMENT 3}

In Experiment 3, we directly compared the effects of presenting saline or coffee solution, either before or after lemon, on habituation of neophobia to lemon. Based on the results of Experiments 1 and 2, our expectation was that saline presented either before or after lemon would disrupt habituation to lemon more than would coffee (or no distractor). To ensure that all groups drank equal amounts of target and distractor solutions throughout habituation training, a fixed quantity of each was presented on each trial.

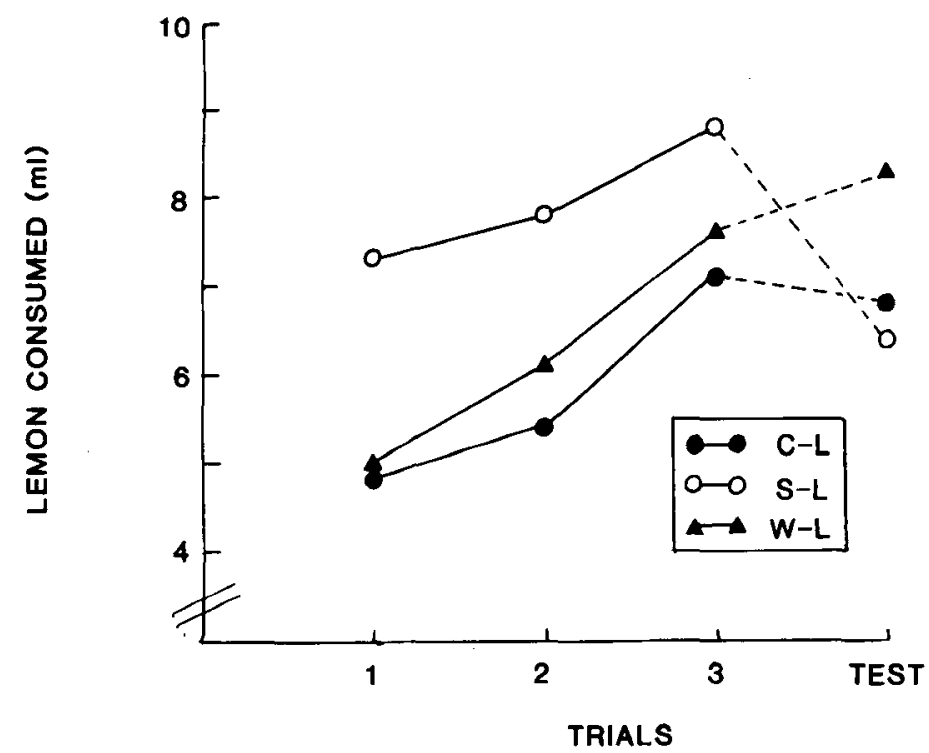

Figure 2. Results of Experiment 2. Consumption of the lemon solution on each day of habituation training and on the final test day. 


\section{Method}

Subjects and Apparatus. The subjects were $\mathbf{5 0}$ male Lister rats of the same age and with the same prior history as those used in Experiments 1 and 2 . The apparatus was the same, except that the calibrated drinking tubes used in pretraining and final testing had glass rather than metal nozzles. From the rats' point of view these glass nozzles were identical to the 5-and 2-ml glass tubes in which the target and distractor solutions were presented during habituation training.

Procedure. After 3 days of pretraining, the rats were asssigned to one of five groups (10 per group) matched for consumption of water on Day 3. Throughout the 3 days of habituation training, all groups received $5 \mathrm{ml}$ of $3 \%$ lemon solution each day. Group L received no distractor; Groups S-L and C-L drank $2 \mathrm{ml}$ of $3 \%$ saline and $1.5 \%$ coffee, respectively, immediately before their access to lemon each day; Groups L-S and L-C drank $2 \mathrm{ml}$ of saline and coffee, respectively, immediately after lemon each day. On the final test day, all animals were given 10-min access to the lemon solution.

\section{Results and Discussion}

The five groups' consumption of water on Day 3 of pretraining ranged from 10.8 to $11.8 \mathrm{ml}$. Since there are no training data to report, the only results of the experiment are from the final test trial; these are shown in Figure 3. On the basis of the results of Experiments 1 and 2, it is apparent that our hypothesis was confirmed: saline acted as a rather more effective distractor than did coffee, whether presented before or after the lemon solution; there is some suggestion that coffee may have been a more effective distractor when presented before lemon than when presented after, but the difference was not great. We analyzed the test scores by using a series of planned contrasts. Groups L-S and S-L drank significantly less lemon than did Groups L-C and C-L $[F(1,45)=4.61$, $p<.05$ ], but there was no difference between the two groups that received their distractor before the lemon solution and the two that received it after the lemon $(F<1)$ and no interaction between type of distractor and order of presentation $(F<1)$. Finally, there was a significant difference between Group L and Groups L-S and S-L combined $[F(1,45)=11.98, p<.01]$.

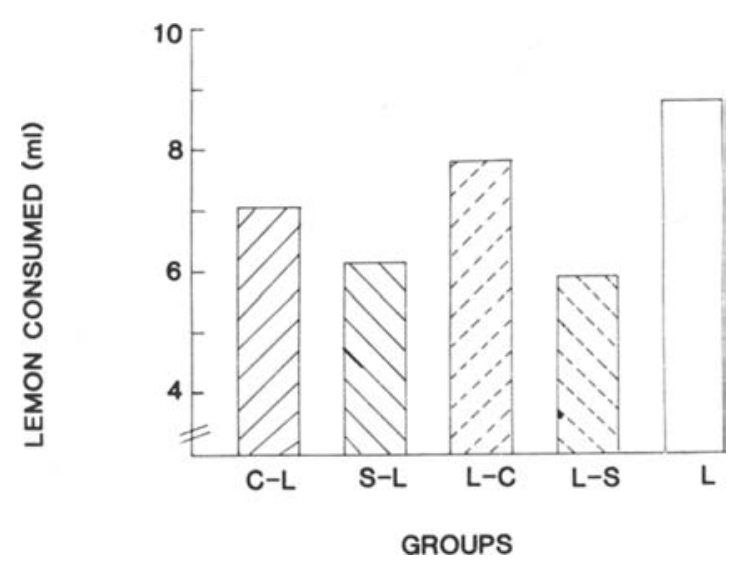

Figure 3. Results of Experiment 3. Consumption of the lemon solution on the final test day.
The presentation of saline, whether before or after lemon, disrupted habituation to lemon more than did the presentation of coffee. Figure 3 suggests, it is true, some parallel with the difference between Experiments 1 and 2: when the distractors were presented after access to lemon (as in Experiment 1), saline had a very much more marked effect than coffee; when they were presented before (as in Experiment 2), the difference seems to have been rather smaller. But there is ample confirmation here of the suggestion that Experiment 2, by failing to control consumption of lemon during training, may have underestimated the extent to which saline before lemon disrupted habituation of neophobia to lemon. In the present experiment, there are no statistical grounds for saying that order of presentation had any effect on the magnitude of distractor effects: saline was a more effective distractor than coffee, regardless of the order of presentation of target and distractor solutions. This conclusion is wholly at variance with Robertson and Garrud's (1983) suggestion that a dissimilar distractor will disrupt habituation more than a similar distractor when it is presented after the target solution, but will be less disruptive when it is presented before the target.

\section{EXPERIMENT 4}

The results of Experiments 1-3 appear to contradict Robertson and Garrud's (1983) conclusions. They are most simply interpreted by saying that a $1.5 \%$ coffee solution is a less effective distractor than a $3 \%$ saline solution not necessarily because it is more similar to the target lemon solution, but because it is a less salient stimulus. No amount of evidence that coffee tastes more like lemon to rats than does saline would be sufficient to prove that this difference in similarity was the critical factor rather than a difference in salience. What is needed is a demonstration that $1.5 \%$ coffee is at least as effective a distractor as $3 \%$ saline when another target solution is used. Experiment 4 tested this proposition.

\footnotetext{
Method

Subjects and Apparatus. The subjects were 24 male hooded Lister rats of the same age and with the same prior history as those used in the previous experiments. The apparatus was the same as that used in Experiment 3. The target solution to which the rats were habituated, referred to as $M$, was a mixture of $1.5 \%$ saline $(\mathrm{NaCl})$ and $5 \%$ sucrose $(\mathrm{w} / \mathrm{v})$ in tap water. The two distractor solutions were the $1.5 \%$ coffee and $3 \%$ saline used in all previous experiments.

Procedure. The rats were given 5 days of pretraining and then

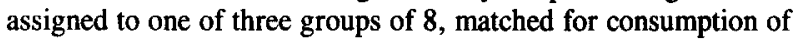
water on the final day. Habituation to the mixture was carried out over 3 days; on the 1st day, all rats received 10-min access to the mixture, but, because they drank a great deal and were thus slow to drink the distractor solution, this was reduced to $5 \mathrm{~min}$ on Days 2 and 3 . Group $M$ animals were returned to their home cages immediately after each habituation trial; Groups M-S and M-C were given $2 \mathrm{ml}$ of the saline and coffee solutions, respectively, before being returned to their home cages. On Day 4, all animals received a 5-min test trial with $\mathrm{M}$.
} 


\section{Results and Discussion}

The three groups' consumption of water on the final day of pretraining was $9.1,8.6$, and $8.8 \mathrm{ml}$ for Groups $\mathrm{M}, \mathrm{M}-\mathrm{S}$, and $\mathrm{M}-\mathrm{C}$, respectively.

On the final test trial, Group $\mathrm{M}$ consumed $10.5 \mathrm{ml}$ of the target mixture, Group M-S, $9.1 \mathrm{ml}$, and Group M-C, $8.5 \mathrm{ml}$. Presentation of either distractor after each habituation trial had disrupted habituation to the mixture, and coffee was, if anything, the more effective distractor. A one-way analysis of variance established that the groups differed on this test trial $[F(2,21)=6.74, p<.01]$, and Newman-Keuls tests showed that Group $M$ consumed more of the mixture than either of the distractor groups, which did not differ from one another.

We can conclude that the $1.5 \%$ coffee solution can act as a no less effective distractor than the $3 \%$ saline solution, and that its failure to disrupt habituation as much as did the saline in Experiments 1-3 cannot be attributed to any general lack of salience.

\section{EXPERIMENT 5}

Given that coffee can act as an effective distractor, it seems plausible to suppose that the critical factor preventing it from disrupting habituation in Experiments 1 to 3 was indeed its similarity to the target solution, lemon. By showing that an aversion conditioned to coffee resulted in a greater reduction in consumption of lemon than of sucrose, Robertson and Garrud (1983) established that the coffee and lemon solutions they used were more similar to one another than to sucrose. Since we have neither copied Robertson and Garrud's procedures nor replicated all their results, it is clearly inappropriate to rely on their data to establish the similarity of the coffee and lemon solutions we used. In Experiment 5, we sought evidence of the similarity of the various solutions we used by conditioning an aversion to either coffee or saline and measuring its generalization to both lemon and the salinesucrose mixture.

\footnotetext{
Method

Subjects and Apparatus. The subjects were 20 male hooded Lister rats from the same stock as those used in previous experiments. Before the start of the present experiment they had all had the experience of drinking water in the apparatus and had also been exposed to each of the four solutions used. The apparatus and solutions were exactly the same as those used in Experiments 3 and 4: lemon, coffee, saline, and the mixture of saline and sucrose.

Procedure. The animals were given two further sessions of 10-min exposure to water, and on the next day were divided into two groups of 10 , matched for their consumption of water. Group $\mathrm{C}+$ was given 10-min access to coffee solution and Group S+ was given 10 -min access to saline solution. They were returned to their home cages for $10 \mathrm{~min}$, and then given a $10-\mathrm{ml} / \mathrm{kg}$ i.p. injection of $.15 \mathrm{M}$ lithium chloride before being finally returned to their home cages to recover. The next day, they were returned to the apparatus and allowed 10 -min access to water. On the following day, they received a second conditioning trial: 10 -min access to the same solution as on the first trial was followed by an injection of lithium chloride. This second conditioning trial was followed by another recovery day, on which the animals were given $10-\mathrm{min}$
}

access to water in the apparatus, and then by two test sessions that were also separated by a water recovery day. On the first test, all animals were given 10-min access to the lemon solution; on the second test, they were given $10-\mathrm{min}$ access to the saline-sucrose mixture.

\section{Results}

Although two conditioning trials were given, since all animals had received prior exposure to the conditioning solutions, the first conditioning trial alone was sufficient to establish an aversion to the solution: all animals in both groups drank less on their second conditioning trial than they had on their first. The means for Trials 1 and 2 were 3.60 and $0.93 \mathrm{ml}$ in Group $\mathrm{C}+$ and 1.78 and $0.45 \mathrm{ml}$ in Group S+.

If lemon is more similar to coffee than to saline, and the sucrose-saline mixture is more similar to saline than to coffee, we should expect that animals conditioned to coffee would show the greater generalized aversion to lemon and that those conditioned to saline would show the greater generalized aversion to the mixure. The results bear this out. Consumption of lemon on the first test trial was $4.61 \mathrm{ml}$ for Group C+ and 5.54 for Group S+; on the second test trial, however, Group $\mathrm{C}+$ consumed $14.86 \mathrm{ml}$ of the mixture, whereas Group $\mathrm{S}+$ consumed only $12.52 \mathrm{ml}$. An analysis of variance revealed a significant overall difference in the consumption of lemon and mixture $[F(1,18)=420.49, p<.01]$, but more importantly a significant interaction between conditioned and test flavors $[F(1,18)=15.14, p<.01]$. The former difference presumably reflects some combination of a preference for the mixture over the lemon solution and the fact that all animals were tested with the lemon before the mixture. The significant interaction implies that the two conditioned flavors did not result in equal generalized aversion to the test flavors. Separate $t$ tests established that Group $\mathrm{C}+$ drank less lemon than Group S+ $(t=2.22)$ but more of the sucrose-saline mixture $(t=3.42, p<05$, in both cases). There was greater generalization to lemon from coffee than from saline, but less generalization to the mixture.

\section{CONCLUSIONS}

The results of the present experiments do not seem very complicated. As others have found before, habituation of rats' neophobia to a target solution was disrupted by presentation of a distractor solution immediately after each habituation trial. The magnitude of this distractor effect was a function of the nature of the target and distractor. Like Robertson and Garrud (1983), we found little disruption of habituation to lemon solution when coffee was the distractor, but a substantial disruptive effect when the distractor was a saline solution. This was not a consequence of any difference in salience between coffee and saline, since, with a different target solution, the results of Experiment 4 established that coffee was at least as effective as saline in disrupting habituation. It is most plausibly attributed to the perceived similarity between lemon 
and coffee solutions (Experiment 5; see also Robertson \& Garrud, 1983, Experiment 5).

Experiments 2 and 3 showed that a distractor presented before the target lemon solution on each habituation trial also disrupted habituation. But, in contrast to Robertson and Garrud's (1983) results, there was no suggestion of a reversal of the relative effectiveness of coffee and saline distractors. Whether they were presented before or after the lemon, saline disrupted habituation more than did coffee.

These results do not seem very surprising and do not require the application of Wagner's $(1976,1981)$ theory for their explanation. They are most simply interpreted as evidence of generalization of habituation between distractor and target solutions. The greater the similarity between the two solutions, the greater the generalization between them, and the more likely it will be that presentation of the distractor should result in generalized habituation to the target. In the limiting case in which distractor and target are indistinguishable, presentation of the distractor, before or after the target, could only increase habituation to the target.

There is another factor that may have contributed to our results, one that is surely relevant to an understanding of distractor effects in general. The simplest explanation of such effects is in terms of generalization decrement. Consumption of the target solution on a test trial must depend on its being perceived as the same solution as that experienced on habituation trials. But since common experience suggests that tastes interact with one another, the presentation of a distractor, whether immediately before or immediately after the target, probably alters the flavor of the target stored in memory, and thus prevents animals from recognizing the solution presented on the test trial as being the same as that to which they had habituated. On this account, a dissimilar distractor might be more effective than one that tastes similar to the target, since it might alter the remembered flavor of the target more profoundly: In Experiments 1 to 3, therefore, the lemon presented alone on the test trial will have, in effect, been a more novel flavor for animals whose previous consumption of it had been preceded or followed by saline, and their neophobia to it would have been restored.

As we have already noted, the present experiments were not exact replications of those reported by Robertson and Garrud (1983). We used somewhat different flavors (saline instead of sucrose and a marginally stronger coffee solution), several habituation trials rather than one, and a 24-h interval between trials rather than a 6 -h one. It is conceivable that one or another of these differences is responsible for the difference in outcome between our experiments and theirs. But it is important to note the similarities as well as the differences between our results: Like them, we found that a dissimilar distractor presented after the target disrupted habituation more than did a similar distractor. The main difference is that we found a similar effect when the distractors were presented before the target, whereas Robertson and Garrud found the opposite pattern of results. There are two features of Robertson and Garrud's results that incline us to question the reliability of the reversal they observed. First, they did not, as we did in Experiment 3, compare the effects of order of presentation of target and similar and dissimilar distractors in a single experiment; their two sets of results were obtained in separate experiments. Second, those results were obtained with different target and distractor solutions: In their Experiment 1, in which a similar distractor presented after the target failed to disrupt habituation, the target was lemon and the distractor was coffee; in their Experiment 6, in which a similar distractor presented before the target significantly disrupted habituation, the target was coffee and the distractor was lemon.

\section{REFERENCES}

Green, K. F., \& PARKer, L. A. (1975). Gustatory memory: Incubation and interference. Behavioral Biology, 13, 359-367.

RoBERTSON, D., \& GARRUD, P. (1983). Variable processing of flavors in rat STM. Animal Leaming \& Behavior, 11, 474-482.

Shanks, D. R., Preston, G. C., \& Stanhope, K. J. (1986). Effects of distractor familiarity on habituation of neophobia. Animal Leaming \& Behavior, 14, 393-397.

Thompson, R. F., \& SPEncer, W. A. (1966). Habituation: A model phenomenon for the study of neuronal substrates of behavior. Psychological Review, 173, 16-43.

Wagner, A. R. (1976). Priming in STM: An information-processing mechanism for self-generated or retrieval-generated depression in performance. In T. J. Tighe \& R. N. Leaton (Eds.), Habituation: Perspectives from child development, animal behavior, and neurophysiology (pp. 95-128). Hilldale, NJ: Erlbaum.

WAGNER, A. R. (1981). SOP: A model of automatic memory processing in animal behavior. In N. E. Spear \& R. R. Miller (Eds.), Information processing in animals: Memory mechanisms (pp. 5-47). Hillsdale, NJ: Erlbaum.

(Manuscript received November 25, 1986; revision accepted for publication May 1, 1987.) 